\title{
Non-culprit ruptured vulnerable plaque healing and stabilization by an aggressive lipid-lowering therapy
}

\author{
Keisuke Shoji $^{1}$ D $\cdot$ Noriyuki Wakana $^{1} \cdot$ Kan Zen $^{1} \cdot$ Satoaki Matoba ${ }^{1}$ \\ Received: 15 February 2021 / Accepted: 17 February 2021 / Published online: 1 March 2021 \\ (c) The Author(s) 2021
}

An 80-year-old man with ST-segment elevation myocardial infarction underwent primary percutaneous coronary intervention (PCI) for $99 \%$ stenosis of the proximal right coronary artery. He underwent a successful PCI with drugeluting stent implantation under the guidance of near-infrared spectroscopy intravascular ultrasound (NIRS-IVUS). However, non-culprit ruptured plaques were identified distal to the culprit lesion (Fig. 1A). The maximum 4-mm lipid core burden index $\left(\operatorname{maxLCBI}_{4 \mathrm{~mm}}\right)$ of the lesion was 743 . Moreover, optical coherence tomography (OCT) revealed a disrupted fibrous cap with a residual lipid-rich plaque (LRP). The minimum lumen area (MLA) was $4.4 \mathrm{~mm}^{2}$ (Fig. 1A). An aggressive lipid-lowering therapy (10 mg rosuvastatin, $10 \mathrm{mg}$ ezetimibe, and proprotein convertase subtilisin/kexin type 9 (PCSK9) inhibitor) lowered the low-density lipoprotein cholesterol levels from 171 to $<17 \mathrm{mg} \mathrm{dL}^{-1}$. One-year follow-up using NIRS-IVUS and OCT revealed a significant maxLCBI ${ }_{4 \mathrm{~mm}}$ decrease (126), a minimum fibrous cap thickness increase, disrupted fibrous cap disappearance, and ruptured plaque healing with an expanding MLA $\left(10 \mathrm{~mm}^{2}\right)$ (Fig. 1B).
Previous intravascular imaging studies reported on the presence of plaque ruptures in both culprit and non-culprit lesions in patients with acute coronary syndrome (ACS). $[1,2]$ Non-culprit plaque ruptures were associated with a fibroatheroma comprising a residual necrotic core. However, there were no major adverse events in patients treated with medical therapy, including statins [1]. In contrast, subclinical ruptured plaques were associated with a high rate of 1-year revascularization [2].

In our patient, NIRS-IVUS and OCT revealed morphological details and drastic changes of the ruptured nonculprit plaque with a residual LRP. A combination of an aggressive lipid-lowering therapy, consisting of a strong statin and a PCSK9 inhibitor, might have healed and stabilized the non-culprit vulnerable ruptured plaques, without significant stenosis.

These imaging findings support the possibility of administering lipid-lowering therapy for the healing and stabilization of non-culprit ruptured plaques and provide historical evidence for its clinical benefits.
Keisuke Shoji

shjksk@koto.kpu-m.ac.jp

1 Kyoto Prefectural University of Medicine, Kyoto, Japan 


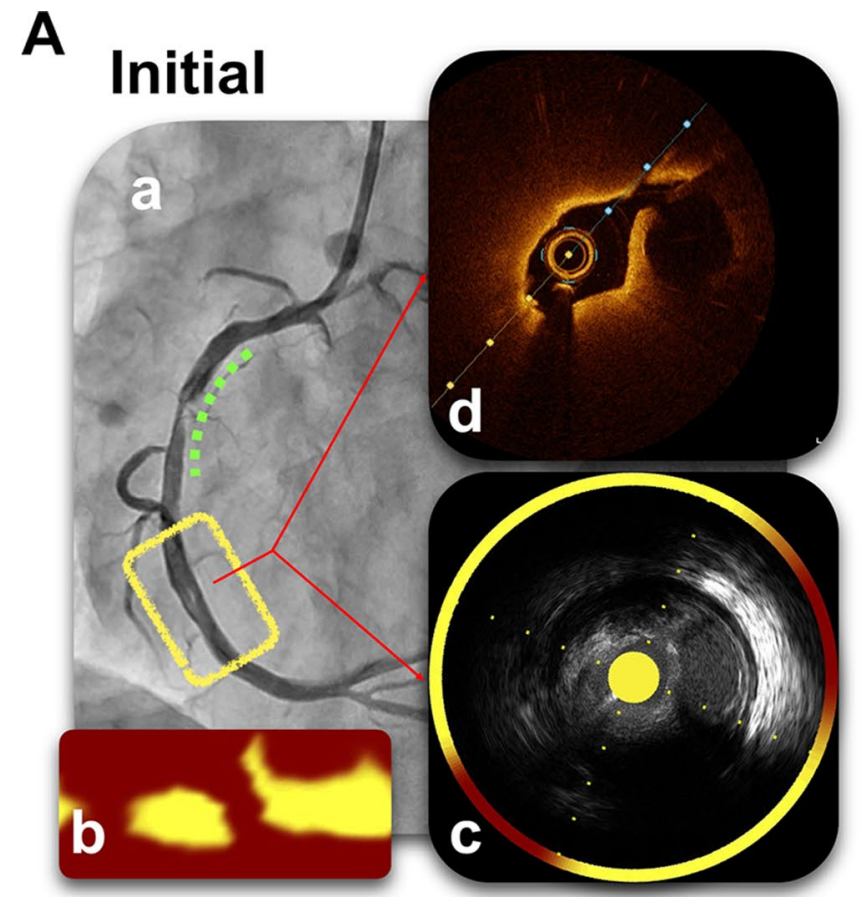

Fig. 1 Drastic changes in the coronary images of a ruptured nonculprit lesion. A Initial coronary images: a coronary angiography, $\mathbf{b}$ and $\mathbf{c}$ initial NIRS-IVUS images, and $\mathbf{d}$ Initial OCT image of the non-culprit lesion. B One-year follow-up coronary images: e followup coronary angiography, $\mathbf{f}$ and $\mathbf{g}$ follow-up NIRS-IVUS images, and $\mathbf{h}$ follow-up OCT image of the non-culprit lesion. Green dotted lines denote the culprit lesions; yellow lines denote the non-culprit

Author contributions KS and NW managed the patient and wrote the manuscript and figures. All authors read and approved the final manuscript.

\section{Funding None.}

Data availability Not applicable.

Code availability Not applicable.

\section{Declarations}

Conflict of interest The author has no conflict of interest.

Ethical approval All procedures performed in this study were in accordance with the ethical standards of the institutional and/or national research committee and with the 1964 Helsinki Declaration and its later amendments or with comparable ethical standards.

Open Access This article is licensed under a Creative Commons Attribution 4.0 International License, which permits use, sharing, adaptation, distribution and reproduction in any medium or format, as long as you give appropriate credit to the original author(s) and the source, provide a link to the Creative Commons licence, and indicate if changes were made. The images or other third party material in this article are

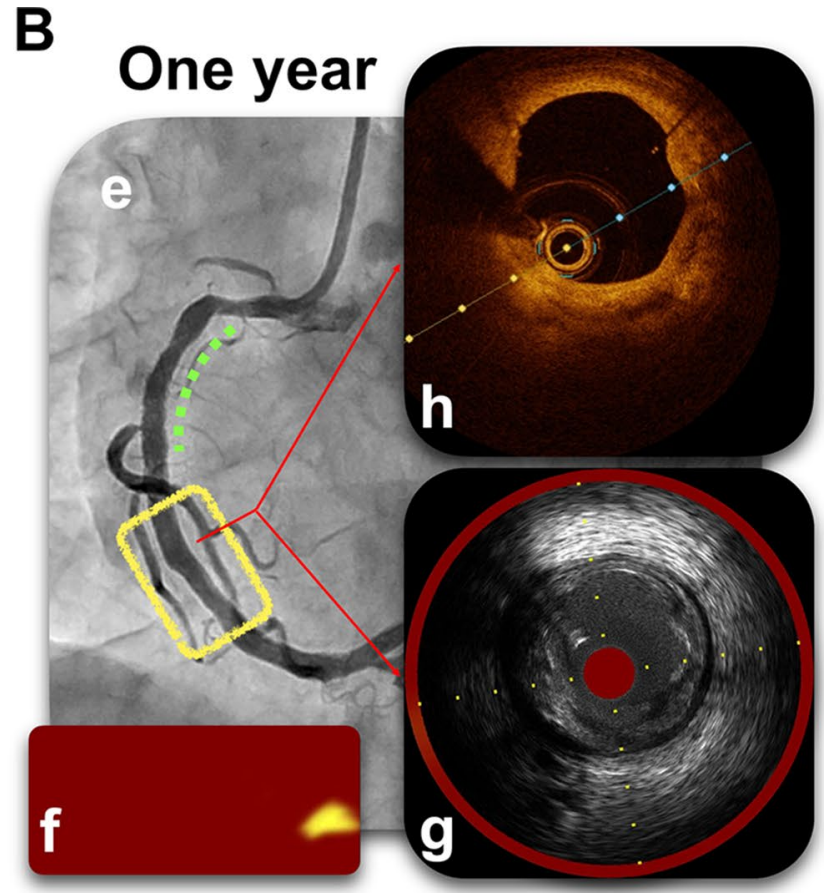

lesions. The maxLCBI ${ }_{4 \mathrm{~mm}}$ in the non-culprit lesion has significantly decreased in the follow-up NIRS-IVUS analysis (from 743 to 126) (b, f). The disrupted fibrous cap in the non-culprit lesion has disappeared

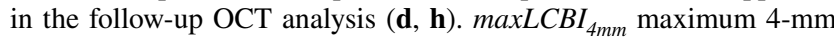
lipid core burden index, NIRS-IVUS near-infrared spectroscopy intravascular ultrasound, $O C T$ optical coherence tomography

included in the article's Creative Commons licence, unless indicated otherwise in a credit line to the material. If material is not included in the article's Creative Commons licence and your intended use is not permitted by statutory regulation or exceeds the permitted use, you will need to obtain permission directly from the copyright holder. To view a copy of this licence, visit http://creativecommons.org/licenses/by/4.0/.

\section{References}

1. Xie Y, Mintz GS, Yang J, Doi H, Iñiguez A, Dangas GD, Serruys PW, McPherson JA, Wennerblom B, Xu K et al (2014) Clinical outcome of nonculprit plaque ruptures in patients with acute coronary syndrome in the PROSPECT study. JACC Cardiovasc Imaging 7:397-405. https://doi.org/10.1016/j.jcmg.2013.10.010

2. Vergallo R, Uemura S, Soeda T, Minami Y, Cho JM, Ong DS, Aguirre AD, Gao L, Biasucci LM, Crea F et al (2016) Prevalence and predictors of multiple coronary plaque ruptures: in vivo 3 -vessel optical coherence tomography imaging study. Arterioscler Thromb Vasc Biol 36:2229-2238. https://doi.org/10.1161/ ATVBAHA.116.307891

Publisher's Note Springer Nature remains neutral with regard to jurisdictional claims in published maps and institutional affiliations. 